\title{
The inflammatory profile of chronic kidney disease patients
}

\section{Hanen Chaker ${ }^{1}$, Faiçal Jarraya ${ }^{1}$, Salma Toumi ${ }^{1 *}$, Khawla Kammoun ${ }^{1}$, Hichem Mahfoudh ${ }^{1}$, Fatma Ayadi ${ }^{2}$, Soumaya Yaich ${ }^{1}$ and Mohamed Ben Hmida ${ }^{1}$}

\begin{abstract}
'Department of Nephrology, Faculty of Medicine, Hedi Chaker University Hospital, University of Sfax \& Laboratory of Research of Renal Pathology LR19ES11, Faculty of Medicine, University of Sfax, Sfax, Tunisia

2Laboratory of research "Molecular Basis of Human Diseases", LR19ES13, Faculty of Medicine, University of Sfax, Sfax, Tunisia; Laboratory of Biochemistry, University of Sfax \& Habib Bourguiba Hospital, Sfax, Tunisia
\end{abstract}

\section{Abstract}

Background: Chronic kidney disease is a worldwide public health issue which is associated with an increased risk of end-stage renal failure and cardiovascular disease. Systemic inflammation exists during chronic renal failure. Recent researches have highlighted the pivotal role of inflammation between renal and cardiovascular disease. The aim of our study is to determine the inflammatory profile of the patient suffering from chronic kidney disease and the influence of hemodialysis on this profile.

Methods: We carried out a cross sectional study on 93 patients in the Nephrology Department at Hedi Chaker University Hospital, Sfax, South of Tunisia. Among those patients, 72 patients underwent hemodialysis and 21 patients had chronic kidney disease at stage 3 . Clinical data and antecedents were collected. Biological samples were taken after informing the patients and taking their consent. Biological data consisted in lipid profile, albumin rate, hemoglobin rate, uric acid concentration and the usual markers of inflammation noting sedimentation rate, C - reactive protein and orosomucoid.

Results: Hemodialysis group of the 72 patients had mean hemodialysis vintage of $54.6 \pm 43$ months The inflammatory profile was worse in hemodialysis patients compared to chronic kidney disease patients. Both sedimentation rate, $\mathrm{C}$ - reactive protein and orosomucoid were higher in hemodialysis group than in chronic kidney disease group with $71 \pm 35.3 \mathrm{~mm}$ vs. $42.1 \pm 15.5 \mathrm{~mm}(p<0.05) ; 14.6 \pm 28.7 \mathrm{mg} / \mathrm{l}$ vs. $6.7 \pm$ $8 \mathrm{mg} / \mathrm{l}(p=0.02) ; 1.3 \pm 0.7 \mathrm{~g} / \mathrm{l}$ vs. $0.9 \pm 0.4 \mathrm{~g} / \mathrm{l}(p=0.01)$, respectively.

Conclusion: Inflammation increases in dialysis patient. It deserves the nephrologist's consideration in order to minimize its harmful effects. The monitoring of inflammation markers must be integrated into the nephrologist's medical practice.

\section{More Information}

*Address for Correspondence: Salma Toumi, Department of Nephrology, Faculty of Medicine, Hedi Chaker University Hospital, University of Sfax \& Laboratory of Research of Renal Pathology LR19ES11, Faculty of Medicine, University of Sfax, Sfax, Tunisia, Email: toumisalma@rocketmail.com; hanen.chaker@yahoo.fr

Submitted: November 10, 2019 Approved: December 16, 2021 Published: December 20, 2021

How to cite this article: Chaker $\mathrm{H}$, Jarraya $\mathrm{F}$, Toumi S, Kammoun K, Mahfoudh H, et al. The inflammatory profile of chronic kidney disease patients. J Clini Nephrol. 2021; 5: 107-111.

DOI: 10.29328/journal.jcn.1001083

Copyright License: (c) 2021 Chaker $\mathrm{H}$, et al. This is an open access article distributed under the Creative Commons Attribution License, which permits unrestricted use, distribution, and reproduction in any medium, provided the original work is properly cited.

Keywords: Inflammation; Chronic kidney disease; Dialysis; C-reactive protein; Markers of inflammation

Check for updates

OPEN ACCESS

\section{Introduction}

Chronic kidney disease (CKD) is a worldwide public health issue. Its prevalence is increasing with life expectancy. Patients with CKD have serious risks of end-stage renal disease and cardiovascular disease (CVD) [1,2]. The mortality risk at the end-stage renal disease is higher than that at the early stage of chronic kidney disease. Moreover, several risk factors are involved ranging from classical ones, such as hypertension, diabetes, dyslipidemia to non-classical ones like inflammation and malnutrition [3]. Actually, chronic kidney disease is characterized by the presence of a systemic low grade of inflammation. Recent researches have highlighted the role of the inflammation in inducing the links between cardiovascular and kidney disease [4]. Inflammation plays an integral role in accelerated atherosclerosis. Systemic micro inflammation determines the extent of vascular endothelium damage, which is the key risk factor for both acute coronary syndrome and chronic kidney disease progression [1].

Furthermore, there is a graded and independent correlation between the glomerular filtration rate and cardiovascular disease outcomes [1]. This mutual interaction between the endothelial function, the heart function and the kidney function is owed to several factors such as hemodynamic conditions, medications, comorbidities and inflammatory mediators.

There are multiple markers with various kinetic models: 
markers whose serum level is multiplied by a factor of 100 such as $\mathrm{C}$ - reactive protein (CRP), markers whose serum level is multiplied by a factor of 2 to 4 such as the orosomucoide, fibrinogen, haptoglobin and markers whose serum level is multiplied by a factor of 0.5 such as ceruloplasmin. Some markers are used in routine practice while others are rarely checked.

The first aim of our study is to determine the inflammatory profile of the patient with chronic kidney disease and the influence of hemodialysis on this profile. The second objective is to determine the most appropriate marker of inflammation that can be reliable and easy to dose and can lead to practical monitoring of the inflammatory status of the dialysis patient.

\section{Methods}

We carried out a cross sectional cohort study on 93 patients. Among those patients, 72 were on hemodialysis and were treated in the Hemodialysis Unit of Hedi Chaker University Hospital, Sfax, Tunisia and 21 patients suffered from chronic kidney disease at stage 3 . All enrolled in this study. Clinical data and antecedents such as the presence of hypertension, diabetes, chronic inflammatory disease, sedentary lifestyle, cerebral stroke, myocardial infraction, vascular calcifications...etc, were collected.

Biological samples were taken after informing and obtaining the patient's consent. Biological data consisted in lipid profile: total cholesterol, high-density lipoprotein (HDL), low-density lipoprotein (LDL), triglycerides (TG), apolipoprotein A (Apo A), apolipoprotein B (Apo B); albumin rate, hemoglobin rate, uric acid concentration as well as the usual markers of inflammation noting sedimentation rate (SR; mm H1), C reactive protein (CRP; $\mathrm{mg} / \mathrm{l}$ ), orosomucoide $(\mathrm{g} / \mathrm{l})$, haptoglobin $(\mathrm{g} / \mathrm{l})$ and fibrinogen $(\mathrm{g} / \mathrm{l})$.

Blood samples were taken using venipuncture from patients with chronic kidney disease or using access points before the beginning of the hemodialysis session from hemodialysis patients.

For statistical study, we used ANOVA test for continuous variables and Chi-square for categorical parameters. A $p$ value $<0.05$ was considered to be statistically significant.

\section{Results}

A group of 93 patients were enrolled in this study. Their mean age was $50.6 \pm 16.9$ years old ( $20-78$ years). The hemodialysis patients were younger than those with chronic kidney disease $(48.3 \pm 16.3$ vs. $58.3 \pm 16.3$ years $)$. The hemodialysis group of 72 patients have mean hemodialysis vintage of $54.6 \pm 43$ months. The group of 21 patients had chronic kidney disease at the third stage with a glomerular filtration rate between 30 and $60 \mathrm{ml} / \mathrm{mn}$. Both groups were comparable for sex, lifestyle, comorbidities and target organs except the abolition of pedal pulses which was more marked in the chronic kidney disease group as shown in Table 1.
The lipid profile including total cholesterol, both LDL and HDL cholesterol, triglycerides, Apo A and Apo B, together with albumin rate, hemoglobin, uric acid rate and body mass index (BMI) are all shown in Table 2.

The levels of total cholesterol noting LDL and HDL cholesterol and also triglycerides were lower in hemodialysis patients than in chronic kidney disease patients $(p<0.05$; $p=0.02 ; p<0.05 ; p=0.02$, respectively). The levels of ApoB were low in dialysis patients compared to chronic kidney disease patients $(0.72 \pm 0.25 \mathrm{~g} / \mathrm{l} v s .0 .88 \pm 0.29 \mathrm{~g} / \mathrm{l} ; p=0.04)$. Likewise, Hemoglobin levels were also lower in hemodialysis patients than in chronic kidney disease patients $(6.6 \pm 1.3$ $\mathrm{g} / \mathrm{dl} v$ s. $12 \pm 1.7 \mathrm{~g} / \mathrm{dl} ; p<0.05$ ). However, the levels of ApoA were similar in both groups. The inflammatory profile of the cohort study including SR, CRP, orosomucoid, haptoglobin and fibrinogen is presented in Table 3.

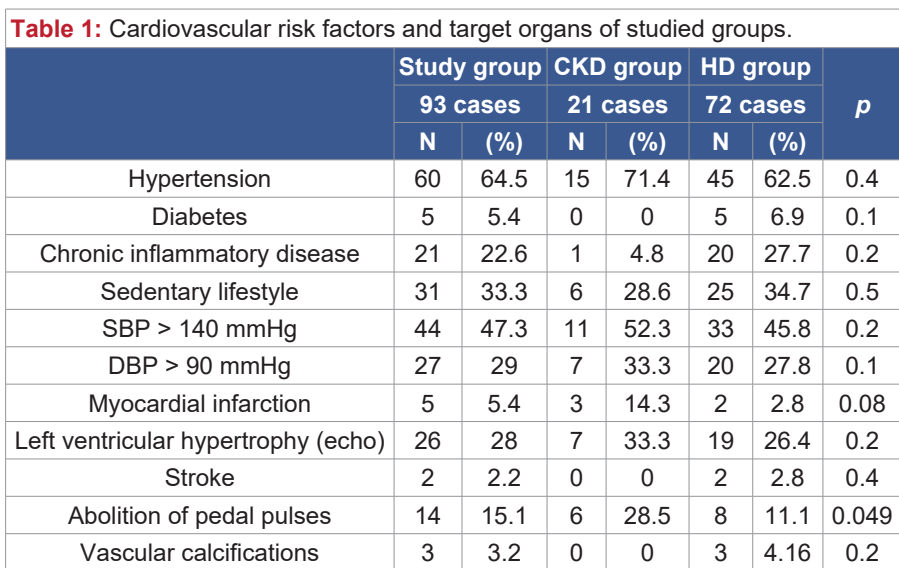
CKD: Chronic Kidney Disease; HD: Hemodialysis; SBP: Systolic Blood Pressure; DBP: Diastolic Blood Pressure

\begin{tabular}{|c|c|c|c|c|}
\hline \multirow{2}{*}{ Table 2: Clinical and biological data of studied groups. } \\
\hline & Study group & CKD group & HD group & \multirow{2}{*}{} \\
\cline { 2 - 5 } & $\mathbf{9 3}$ cases & $\mathbf{2 1}$ cases & $\mathbf{7 2}$ cases & \\
\hline Total cholesterol $(\mathrm{mmol} / \mathrm{l})$ & $4.6 \pm 1.3$ & $5.1 \pm 1.1$ & $4.2 \pm 1.2$ & $<0.05$ \\
\hline HDL $(\mathrm{mmol} / \mathrm{l})$ & $1 \pm 0.3$ & $1.2 \pm 0.2$ & $0.8 \pm 0,2$ & 0.02 \\
\hline LDL $(\mathrm{mmol} / \mathrm{l})$ & $2.8 \pm 0.9$ & $3.5 \pm 0.8$ & $2.5 \pm 0,8$ & $<0.05$ \\
\hline Triglycerides $(\mathrm{mmol} / \mathrm{l})$ & $1.6 \pm 0.7$ & $1.9 \pm 0.7$ & $1.5 \pm 0.7$ & 0.02 \\
\hline Apo A $(\mathrm{g} / \mathrm{l})$ & $1.2 \pm 0.2$ & $1.2 \pm 0.2$ & $1.2 \pm 0.3$ & 0.3 \\
\hline Apo B $(\mathrm{g} / \mathrm{l})$ & $0.7 \pm 0.2$ & $0.8 \pm 0.2$ & $0.7 \pm 0.2$ & 0.04 \\
\hline Albumin rate $(\mathrm{g} / \mathrm{l})$ & - & - & $35.9 \pm 6.9$ & - \\
\hline Hemoglobin rate $(\mathrm{g} / \mathrm{dl})$ & $7.8 \pm 2.6$ & $12 \pm 1.7$ & $6.6 \pm 1.3$ & $<0.05$ \\
\hline Uric acid $(\mu \mathrm{mol} / \mathrm{l})$ & $436.6 \pm 92.2$ & $452 \pm 87.5$ & $434.6 \pm 93.3$ & 0.6 \\
\hline BMl $\left(\mathrm{Kg} / \mathrm{m}^{2}\right)$ & $21.4 \pm 4.6$ & $25.2 \pm 6.2$ & $20.2 \pm 3.3$ & 0.07 \\
\hline
\end{tabular}

CKD: Chronic Kidney Disease; HD: Hemodialysis; HDL: High-Density Lipoprotein; LDL: Low-Density Lipoprotein; ApoA: Apolipoprotein A; ApoB: Apolipoprotein B; BMI: Body Mass Index

Table 3: Inflammatory markers of studied groups.

\begin{tabular}{|c|c|c|c|c|}
\hline & $\begin{array}{c}\text { Study group } \\
\text { Table 3: Inflammatory markers of studied groups. }\end{array}$ & CKD group & HD group & $\boldsymbol{P}$ \\
\hline SR $(\mathrm{mm} \mathrm{H} 1)$ & $64.5 \pm 34.1$ & $42.1 \pm 15.5$ & $71 \pm 35.3$ & $<0.05$ \\
\hline CRP $(\mathrm{mg} / \mathrm{l})$ & $12.8 \pm 25.6$ & $6.7 \pm 8$ & $14.6 \pm 28.7$ & 0.02 \\
\hline Orosomucoid $(\mathrm{g} / \mathrm{l})$ & $1.2 \pm 0.7$ & $0.9 \pm 0.4$ & $1.3 \pm 0.7$ & 0.01 \\
\hline Haptoglobin $(\mathrm{g} / \mathrm{l})$ & $1.8 \pm 1.3$ & $1.3 \pm 0.8$ & $1.9 \pm 1.4$ & 0.08 \\
\hline Fibrinogen $(\mathrm{g} / \mathrm{l})$ & $4.7 \pm 1.4$ & $4.5 \pm 0.7$ & $4.7 \pm 1.9$ & 0.5 \\
\hline Serum ferritin $(\mathrm{ng} / \mathrm{ml})$ & - & - & $190.9 \pm 259.8$ & - \\
\hline Serum iron $(\mu \mathrm{mol} / \mathrm{l})$ & - & - & $15.8 \pm 9.7$ & - \\
\hline $\begin{array}{l}\text { CKD: Chronic Kidney Disease; HD: Hemodialysis; SR: Sedimentation Rate; } \\
\text { CRP: C - Reactive Protein }\end{array}$ & & & \\
\hline
\end{tabular}


The results of our study showed that a low grade of inflammation was present in patients with chronic kidney disease as CRP levels reach $12.8 \pm 25.6 \mathrm{mg} / \mathrm{l}$ in the studied population. These levels were more important in the hemodialysis group compared to the group of patients with the third stage of chronic kidney disease $(14.6 \pm 28.7 \mathrm{mg} / \mathrm{l} v \mathrm{~s}$. $6.7 \pm 8 \mathrm{mg} / \mathrm{l} ; p=0.02$ ).

The inflammatory profile was worse in the hemodialysis group than in the chronic kidney disease group. All SR, CRP and Orosomucoid were higher in the hemodialysis group than in the chronic kidney disease group with $71 \pm 35.3 \mathrm{~mm}$ vs. $42.1 \pm 15.5 \mathrm{~mm}(p<0.05) ; 14.6 \pm 28.7 \mathrm{mg} / \mathrm{l}$ vs. $6.7 \pm 8$ $\mathrm{mg} / \mathrm{l}(p=0.02) ; 1.37 \pm 0.76 \mathrm{~g} / \mathrm{l}$ vs. $0.92 \pm 0.44 \mathrm{~g} / \mathrm{l}(p=0.01)$, respectively (Table 3 ).

\section{Discussion}

Systemic inflammation is a common feature in the uremic phenotype and it is associated with bad outcomes. The awareness about the importance of inflammation assessment in CKD patients has risen in recent years, and despite the development of novel biomarkers, C-reactive protein is still the most measured inflammatory parameter [5].

Our study showed that a low grade of inflammation was present in patients with chronic kidney disease and was accentuated when hemodialysis was launched. Thus, monitoring this inflammation was crucial.

The sedimentation rate is known as a weak inflammation marker in patients with chronic kidney disease, as its value correlates with the degree of anemia that greatly exists in these patients. In our study, the SR value was $71 \pm 35.3$ $\mathrm{mm}$ in hemodialysis patients but it was of $42.1 \pm 15.5 \mathrm{~mm}$ in patients at the third stage of chronic kidney disease $(p<0.05)$. The hemoglobin levels were $6.66 \pm 1.33 \mathrm{~g} / \mathrm{dl}$ and $12 \pm 1.7 \mathrm{~g} / \mathrm{dl}(p<0.05)$, respectively.

The haptoglobin levels were higher in hemodialysis patients than in the third stage of chronic kidney disease patients having the values $1.99 \pm 1.40$ and $1.34 \pm 0.83$, respectively. Yet, this difference couldn't be considered significant. In fact, haptoglobin was influenced by the degree of hemolysis present in hemodialysis patient. Actually, several hypotheses might occur due to many factors. A case example is tube bending during the hemodialysis session, dialysate impurity, and endotoxins contamination. These factors might contribute to a chronic hemolysis, which might decrease the haptoglobin levels.

The orosomucoid levels were greater in hemodialysis patients compared to the third stage of chronic kidney disease patients, with values $1.37 \pm 0.76$ and $0.92 \pm 0.44$ ( $p=0.01)$, respectively. The orosomucoid or alpha-1-acid glycoprotein is a major positive protein of the inflammatory reaction (with CRP and haptoglobin). Its half-life is from 2 to
5 days. The orosomucoid rises before haptoglobin, and then, it joins the haptoglobin. Besides, the two molecules correlate in their evolution. Hence, orosomucoid is useful for dating inflammation. This parameter is not affected by the degree of anemia, so it can be a reliable inflammation marker in patients with chronic kidney disease.

To sum up, we can state that CRP, orosomucoid and haptoglobin are the major positive proteins of inflammation and together they define a specific inflammatory profile of the dialysis patient. During the dialysis therapy, the chronic inflammation is the rule. However, acute inflammation might occur as a result of surgery or infection. The concomitant elevation of CRP, orosomucoid and haptoglobin gives evidence of this chronic status, especially if albumin is diminished.

The monitoring of this inflammatory profile might be an effective tool in diagnosis and follow up of inflammation. In regard to cost, the simplicity of measurement and the interpretation, CRP remains the most useful marker of inflammation. In accordance with data from the Dialysis Outcomes and Practice Patterns Study (DOPPS) registry, the use of CRP in dialysis units around the world has considerably increased in the last decade [6]. Interestingly enough, the dialysis facilities that reported the measuring of CRP in more than $50 \%$ of their patients had lower cardiovascular related mortality. These findings might be accounted for the rising awareness of potentially treatable conditions that lead to an early diagnosis and intervention [5,7-9].

Inflammation is the main cause that speeds up atherosclerosis. Also, inflammation and lipid abnormalities are interrelated. Our results showed that the lipid profile is more altered in hemodialysis. The total cholesterol value was less in hemodialysis group of patients compared to the chronic kidney disease group $(p<0.05)$. Both LDL and HDL cholesterol diminished. This could be explained by malnutrition observed in hemodialysis patients. The BMI was low in dialysis patients and it was mainly generated by anorexia induced by uremia and anemia.

The HDL cholesterol (protective cholesterol) significantly decreased in hemodialysis, which means that the dialysis patient was more vulnerable to the cardiac attack. The levels of ApoB were lower in dialysis patients compared to the chronic kidney disease group: $0.72 \pm 0.25 \mathrm{~g} / \mathrm{l} v s .0 .88 \pm 0.29$ $\mathrm{g} / \mathrm{l}(p=0.04)$. Yet, the levels of ApoA were similar in both groups.

Apo A1 is the principal apolipoprotein contained in HDL and ApoB is the single molecule in LDL [10]. The levels of ApoB were low in the hemodialysis patients mostly because of malnutrition. These results rejoin the most common MIA syndrome; associated to malnutrition, inflammation and atherosclerosis in the chronic kidney disease; and the uremic patient.

Many studies have shown that the increase of inflammation 
markers already exist at stage 3 and stage 4 of CKD leading to an inflammatory reaction. These abnormalities can lead to serious complications of CKD such as cardiovascular disease, atherosclerosis, malnutrition and anemia. Furthermore, the disease progression and the dialysis treatment can aggravate this inflammatory status $[11,12]$.

In fact, when the chronic kidney disease reaches its final stage, the heart function will be inevitably weakened. Despite the correction of water overload and the purification of little and middle size uremic toxins with dialysis; inflammation profile worsens on dialysis. It is a veritable chronic process in hemodialysis, caused by the dialysate impurity, the membrane bio-incompatibility, the blood interaction with tubes and dialyzer, the sterilization agent such as Ethylen oxyde (ETO), catheter use and failed allografts $[13,14]$. Added to that, it may be intermittent due to a coexisting process such as tooth decay or acute infection.

Patients with end-stage renal disease undergoing chronic hemodialysis (HD) present higher mortality rates compared to the general population. Once patients are on HD, the risk of cardiovascular death is approximately 30 times higher than that in the general population and it remains between 10 and 20 times higher after stratification for age, gender, and the presence of diabetes [4]. The traditional risk factors seem to be inadequate to completely explain the remarkable prevalence of cardiovascular mortality and morbidity observed in the uremic population. Hence, the role for chronic inflammation has been well established in the development of atherosclerotic disease. In fact, the accelerated atherosclerotic process of end-stage renal disease may involve several interrelated processes such as oxidative stress, endothelial dysfunction, vascular calcification and inflammation.

Along with the cardiovascular impact of chronic inflammation, several publications have shown that the loss of renal function is faster if the CRP and tumor necrosis factor- alpha (TNF-alpha) levels are increased [15]. Monitoring these inflammatory markers appears to be important in the followup of patient with chronic kidney disease before and after the end stage renal disease.

Further studies will be necessary to explain the inflammatory status of the hemodialysis patients so as to reduce as much as possible all sources of inflammation. Last but not least, we could be criticized for not considering the age factor in our study. Indeed, the hemodialysis patients had a high degree of inflammation although they were younger than chronic renal disease patients.

\section{Conclusion}

Inflammation increases in dialysis patients. It can be considered as the silent killer of the patient on dialysis therapy. Besides, it deserves the nephrologist's attention in order to minimize its harmful action. Above all, the monitoring of inflammation markers has to be integrated into the nephrologist's medical practice.

Authors' contributions: $\mathrm{HC}, \mathrm{FJ}$ and $\mathrm{HM}$ collecting data for text and writing the first manuscript; FA realized laboratory analysis; ST, KK, SY and MBH reviewing revisions and final manuscript.

\section{References}

1. Udeanu M, Guizzardi G, Di Pasquale G, Marchetti A, Romani F, et al. Relationship between coronary artery disease and C-reactive protein levels in NSTEMI patients with renal dysfunction: a retrospective study. BMC Nephrol. 2014; 15: 152.

PubMed: https://pubmed.ncbi.nlm.nih.gov/25230678/

2. González-Espinoza L, Rojas-Campos E, Medina-Pérez M, PeñaQuintero P, Gómez-Navarro B, et al. Pentoxifylline decreases serum levels of tumor necrosis factor alpha, interleukin 6 and C-reactive protein in hemodialysis patients: results of a randomized double-blind, controlled clinical trial. Nephrol Dial Transplant. 2012; 27: 2023-2028. PubMed: https://pubmed.ncbi.nlm.nih.gov/21968012/

3. Dekker MJE, van der Sande FM, van den Berghe F, Leunissen KML, Kooman JP. Fluid Overload and Inflammation Axis. Blood Purif. 2018; 45: 159-165.

PubMed: https://pubmed.ncbi.nlm.nih.gov/29478061/

4. Panichi V, Scatena A, Migliori M, Marchetti V, Paoletti S, et al. Biomarkers of Chronic Inflammatory State in Uremia and Cardiovascular Disease. Int J Inflamm. 2012; 2012: 360147. PubMed: https://pubmed.ncbi.nlm.nih.gov/22701810/

5. Cobo G, Qureshi AR, Lindholm B, Stenvinkel P. C-reactive Protein: Repeated Measurements will Improve Dialysis Patient Care. Semin Dial 2016; 29: 7-14.

PubMed: https://pubmed.ncbi.nIm.nih.gov/26360923/

6. Combe C. Dose de dialyse, nutrition, inflammation: quelle est la relation? Néphrologie Thérapeutique. 2010; 6: 7-12.

7. Cobo G, Lindholm B, Stenvinkel P. Chronic inflammation in end-stage renal disease and dialysis. Nephrol Dial Transplant. 2018; 33: iii35-40. PubMed: https://pubmed.ncbi.nlm.nih.gov/30281126/

8. Bazeley J, Bieber B, LiY, Morgenstern H, Sequera P de, Combe C, et al. C-Reactive Protein and Prediction of 1-Year Mortality in Prevalent Hemodialysis Patients. Clin J Am Soc Nephrol. 2011; 6: 2452-2461. PubMed: https://pubmed.ncbi.nlm.nih.gov/21868617/

9. Nowak KL, Chonchol M. Does Inflammation Affect Outcomes in Dialysis Patients? Semin Dial. 2018; 31: 388-397. PubMed: https://pubmed.ncbi.nlm.nih.gov/29513906/

10. Kaysen GA, Dalrymple LS, Grimes B, Chertow GM, Kornak J, et al. Changes in serum inflammatory markers are associated with changes in apolipoprotein A1 but not B after the initiation of dialysis. Nephrol Dial Transplant. 2014; 29: 430-437.

PubMed: https://pubmed.ncbi.nlm.nih.gov/24009290/

11. Tbahriti HF, Meknassi D, Moussaoui R, Messaoudi A, Zemour L, et al. Inflammatory status in chronic renal failure: The role of homocysteinemia and pro-inflammatory cytokines. World J Nephrol. 2013; 2: 31-37.

PubMed: https://pubmed.ncbi.nlm.nih.gov/24175263/

12. Babaei M, Dashti N, Lamei N, Abdi K, Nazari F, et al. Evaluation of plasma concentrations of homocysteine, IL-6, TNF-alpha, hs-CRP, and total antioxidant capacity in patients with end-stage renal failure. Acta Med Iran. 2014: 893-898.

PubMed: https://pubmed.ncbi.nlm.nih.gov/25530051/ 
13. Banerjee T, Kim SJ, Astor B, Shafi T, Coresh J, Powe NR. Vascular Access Type, Inflammatory Markers, and Mortality in Incident Hemodialysis Patients: The Choices for Healthy Outcomes in Caring for End-Stage Renal Disease (CHOICE) Study. Am J Kidney Dis. 2014; 64: 954-961.

PubMed: https://pubmed.ncbi.nlm.nih.gov/25266479/

14. Achinger SG, Ayus JC. Inflammation from dialysis, can it be removed?
Nephrol Dial Transplant 2013; 28: 770-773.

PubMed: https://pubmed.ncbi.nlm.nih.gov/23109559/

15. Carlsson AC, Carrero JJ, Stenvinkel P, Bottai M, Barany $P$, et al. High Levels of Soluble Tumor Necrosis Factor Receptors 1 and 2 and Their Association with Mortality in Patients Undergoing Hemodialysis. Cardiorenal Med. 2015; 5: 89-95.

PubMed: https://www.ncbi.nlm.nih.gov/pmc/articles/PMC4427143/ 